\section{Migraine headache in patients with idiopathic intracranial hypertension}

\author{
Farzad Sina, Saeed Razmeh, Neda \\ Habibzadeh, Arefeh Zavari, Mona \\ Nabovvati \\ Iran University of Medical sciences, \\ Tehran, Iran
}

\begin{abstract}
Migraine is a neurological disorder that afflicts many people in the world and can cause severe disability during the attacks. The pathophysiology of migraine is complex and not fully understood. It seems that migraine is common in idiopathic intracranial hypertension (IIH). However, the association between migraine headache and IIH is still unclear. The present study was conducted to assess the prevalence of migraine headache and associated factors in IIH patients. In this cross-sectional study, a total of 68 patients diagnosed with IIH underwent a medical history interview and a neurological examination. The diagnosis of migraine was based on the four diagnostic criteria of the International Classification of Headache Disorders 3rd edition. Forty-five patients $(63.2 \%)$ met the diagnostic criteria of migraine headache. There was no significant difference between patients with and without migraine headache in respect of their age, gender, body mass. This study revealed high prevalence of migraine headache in IIH patients; appropriate treatment can reduce their headache and prevent unnecessary treatments for IIH.
\end{abstract}

\section{Introduction}

Migraine is one of the most common cause of headache and consultation for headache in America, South-East Asia, Europe, and the Western Pacific. The mechanism and pathophysiology of migraine is complex and it is related to environmental and genetic factores. 1,2 It afflicts many people in the world and after puberty is more prevalent in women. The symptoms associated with migraine headache include headache that usually is pulsatile and unilateral, nausea and vomiting, vertigo, photophobia and phonophobia and in migraine with aura, it can associated with visual, sensory, motor and speech symptoms.3-5 Idiopathic intracranial hypertension (IIH) presents with headache, diplopia due to cra- nial nerve palsy, visual obscuration, tinnitus and papilledema with normal cerebrospinal fluid (CSF) analysis and neuroimaging. 6,7 Many of this patient after treatment complain of headache without any abnormality in optic disc and CSF pressure. In present study, we assessed the prevalence of migraine in patients with IIH as common cause of headache.

\section{Materials and Methods}

This cross-sectional study was done in neurology ward of Rasoul-Akram Hospitals affiliated with the Iran University of Medical Sciences, Tehran, Iran. The study was approved by the Ethical Committee of the University. The study population included the patients with intracranial hypertension (IIH) whose disease was under control and without papilledema and raised of CSF pressure. They were interviewed in the neurology clinic. The patients who had uncontrolled diseases, sinus vein thrombosis were excluded. All participants signed a written informed consent. The diagnosis of migraine headache in the patients was based on the four diagnostic criteria of the International Classification of Headache Disorders 3rd edition.8,9 After making the diagnosis of migraine headache, the IIH patients were divided into two groups: those with and without migraine. The Clinical characteristics assessed in both groups were age, gender, duration of disease, BMI index, and family history of migraine. Body weight and height of all participants was obtained from her/his information and BMI was calculated as weight in kilograms divided by the square of height in meter. SPSS 22 was used for statistical analyses and $\mathrm{P}<0.05$ was considered as a significant level. Descriptive statistics including mean and standard deviation (SD) were used to characterize the study population and t-student test were used for comparing quantitative variable between two groups.

\section{Results}

The 68 patients known case of IIH were studied, 11 males $(16.2 \%)$ and 57 females $(83.8 \%)$. The age range was $9-63$ years. the mean age was 33.99 years. The mean BMI of the all patients was $28.6 \pm 4.4 \mathrm{~kg} / \mathrm{m}^{2}$ and the duration of $71 \%$ of IIH patients was under 5 years, $21 \%$ between $5-10$ years and $8 \%$ above the 10 years. The 45 patients $(63.4 \%)$ out of a total of 68 subjects diagnosed with IIH met the migraine diagnostic criteria. There were 37 women $(82.2 \%)$ and
Correspondence: Saeed Razmeh, Iran University of Medical sciences, Tehran, Iran. E-mail: srazmeh@yahoo.com

Key words: Migraine headache; Idiopathic intracranial hypertension; Neurological disorder.

Received for publication: 25 June 2017. Accepted for publication: 5 July 2017.

This work is licensed under a Creative Commons Attribution NonCommercial 4.0 License (CC BY-NC 4.0).

(C) Copyright F. Sina et al., 2017

Licensee PAGEPress, Italy

Neurology International 2017;9:7280

doi:10.4081/or.2017.7280

8 men $(17.8 \%)$. The mean age was $34.8 \pm 12.02$ years, and the mean BMI was $28.4 \pm 4.2 \mathrm{~kg} / \mathrm{m}^{2}$. In migraine population, $75 \%$ of had the disease duration under 5 years and $17.8 \%$ between $5-10$ years and others above the 10 years. The family history of migraine in patients with migraine headache was $68 \%$. The 23 patients [women20 (86.9\%) and 3 men (13.1\%)] were without migraine headache. The mean age was 33.3. The mean BMI in this patient's was $29 \mathrm{~kg} / \mathrm{m}^{2}$ (Table 1) and the disease duration was $61 \%$ under 5 years and $38 \%$ between $5-10$ years and $1 \%$ above the 10 years. The family history of migraine in this patient was $62 \%$. In patients with migraine headache, $33 \%$ had headache more in frontal, $16 \%$ occipital and other had generalized. 51 percent had moderate chronic daily headache and only 5 patients had migraine with aura.

\section{Discussion}

The migraine headache is a common disorder in IIH patients. In our study, there is significant association between the incidence of IIH and migraine headache and the prevalence of migraine was $63.2 \%$. However, evaluation of the association between IIH and migraine headache needs a case control study which compare the migraine prevalence between patients and general population that present study lacks control group. In our study, no differences were found between groups with and without migraine headache in their age, gender and body max index $(\mathrm{P}>0.05)$. In study by Kathleen et al., that assessed the clinical characteristics of IIH patients with and without papilledema, shows that the prevalence of migraine headache in patients with- 
Table 1. Overall information about the patients studied.

\begin{tabular}{lccc} 
Signs and symptoms & Overall patients & With migraine & Without migraine \\
Mean ages (years) & 33.99 & 34.8 & 33 \\
Female (n) & $57(83.8 \%)$ & $37(82.2 \%)$ & $20(86.9 \%)$ \\
\hline Male $(\mathrm{n})$ & $11(16.2 \%)$ & $8(17.8 \%)$ & $3(13.1 \%)$ \\
BMI $\left(\mathrm{kg} / \mathrm{m}^{2}\right)$ & 28.6 & 28.4 & 29 \\
\hline Family history of migraine (\%) & 65 & 68 & 62 \\
\hline
\end{tabular}

BMI, body mass index.

out papilledema was $65 \%$ and in patients with papilledema was $45 \% .{ }^{10}$ Curiously there is relation between migraine and intracranial hypertension that is very complex and need more study. Mathew et al. assessed the prevalence of intracranial pressure in patients with chronic unresponsive migraine headache, in this study 44 patients with chronic migraine headache and stenosis in sinus vein in mri venography were assessed that $86.4 \%$ of this patients have raised intracranial hypertension (csf pressure $>200 \mathrm{mmH} 2 \mathrm{O}$ ). The $77 \%$ of this patient, experience decreased headache with treatment of ICP raising. ${ }^{10}$ In another study by Ramadan that assessed the IIH in the patients with chronic daily headache, 12 patients have intracranial hypertension that do not have any evidence of papilledema, visual obscuration and decreased visual acuity and the treatment with acetazolamide reduce their chronic headache. ${ }^{11}$ However, it seems that in any patients with chronic migraine headache that is refractory and do not respond to usual prophylactic migraine headache, it is essential that done spinal tap for rule out of idiopathic intracranial hypertension without papilledema. ${ }^{12}$ In our study obesity was frequent in IIH patients with and without migraine, the relation between obesity and IIH remains unclear. It is seem that Healthy overweight individuals may have CSF pressure above the normal range and also the obesity associated with increased severity and frequency of migraine attacks. ${ }^{13}$

\section{Conclusions}

In conclusion, as mentioned earlier there is a high prevalence of migraine headache in patients with $\mathrm{IIH}$, and in any patients with IIH who complain of headache after treatment, migraine headache should considered. Appropriate treatment can improve their quality of life and prevent unnecessary treatments for IIH.

\section{References}

1. Victor TW, Hu X, Campbell JC, et al. Migraine prevalence by age and sex in the United States: a life-span study. Cephalalgia 2010;30:1065-72.

2. Diener HC, Charles A, Goadsby PJ, Holle D. New therapeutic approaches for the prevention and treatment of migraine. Lancet Neurol 2015;14:101022.

3. Kavuk I, Yavuz A, Cetindere U, et al. Epidemiology of chronic daily headache. Eur J Med Res 2003;8:23640.

4. MacGregor EA, Rosenberg JD, Kurth T. Sex-related differences in epidemio- logical and clinic-based headache studies. Headache 2011;51:843Y859.

5. Silberstein SD, Saper JR, Freitag F. Migraine: diagnosis and treatment. In: Silberstein SD, Lipton RB, Dalessio DJ, editors. Wolff's headache and other head pain. 7th ed. New York, NY: Oxford University Press; 2001. pp. 121237.

6. Digre KB, Corbett JJ. Idiopathic intracranial hypertension (pseudotumor cerebri): A reappraisal. Neurologist 2001;7:2-67.

7. Mathew NT, Ravishankar K, Sanin LC. Coexistence of migraine and idiopathic intracranial hypertension without papilledema. Neurology 1996;46:122630.

8. Headache Classification Committee of the International Headache Society (IHS). The International Classification of Headache Disorders, 3rd edition (beta version). Cephalalgia 2013;33:629-808.

9 De Simone R, Ranieri A, Montella S, et al. Intracranial pressure in unresponsive chronic migraine. J Neurol 2014;261: 1365-73.

10. Mathew NT, Ravishankar K, Sanin LC. Coexistence of migraine and idiopathic intracranial hypertension without papilledema. Neurology 1996;46:122630.

11. Ramadan NM. Intracranial hypertension and migraine. Cephalalgia 1993;13: 210-1.

12. Digre KB, Nakamoto BK, Warner JE, et al. A comparison of idiopathic intracranial hypertension with and without papilledema. Headache 2009;49:18593.

13. Bigal ME, Liberman JN, Lipton RB. Obesity and migraine: a population study. Neurology 2006;66:545-50. 